\title{
Development of a Process for Perceptual Index Establishment based
}

\section{on Axiomatic Design}

\author{
Yilun Zhang ${ }^{1,2}$, Lichao Peng ${ }^{1,2}$, Jing Shen ${ }^{2}$, Zhang Zhinan ${ }^{1,2}$, Fei Tao ${ }^{3}$ \\ ${ }^{1}$ School of Mechanical Engineering, Shanghai Jiao Tong University, Shanghai \\ 2 Joint Design Innovation Lab of Shanghai Jiao Tong University and CIDI Southwest Center, \\ Chongqing \\ ${ }^{3}$ School of Automation Science and Electrical Engineering, Beijing University
}

\begin{abstract}
:
With the increasingly intense competition in the market, more attention has been paid to customer's perceptual requirements. Combining human sensibility and technology, Kansei Engineering plays a crucial role for industrial designers in making decisions at an earlier design stage in product development. However, sometimes it is challenging to define the customer requirements during the market research phase, especially for the perceptual requirements. Given this problem, this paper develops a process for establishing perceptual indexes based on the axiomatic design approach to improve design efficiency. To achieve this, an initial perceptual index is first built based on data previously collected from designers or design agencies. Then, the theory of axiomatic design is used to classify the schemes. The final perceptual index is filtered and built according to the frequency of occurrence.
\end{abstract}

Keywords: Axiomatic Design, Kansei Engineering, Industrial Design 


\section{Introduction}

With increasingly intense competition in the market, the development of products to meet customer needs has already emerged as a core competency. In the axiomatic design ( $\mathrm{AD}$ ) process, for example, mapping customers' needs from the user domain to the functional domain is the starting point for design (Suh, 2001). However, it is often difficult to accurately define the user requirements or attributes of a product, or even vaguely define them (Suh, 2001) due to the large amounts of perceptual indexes contained in the user domain. As a branch of Kansei Engineering (KE), research on perceptual indexes has been proposed to maximize customers' satisfaction with their purchases (Vieira et al., 2017). To accurately transform customer attributes (CAs) into functional requirements (FRs), the measurement and establishment of perceptual indexes is necessary.

In business activities, the input of a new product design mostly originates from the market requirements documents or specification (MRD or MRS) (Suh, 2001). Generally, an MRD is a report consisting of competitive analysis, market survey, and other information (Samuel, 2009). In the perceptual part, personas are often taken by the enterprise as a solution to define customers' perceptual requirements. However, the personas formed by brainstorming often describe customers' perceptual requirements fuzzily with low cohesion (i.e., the information axiom), and many user elements duplicate the same requirements (in whole or part) with high coupling (i.e., the independence axiom). Therefore, within the same product field, it is necessary to find perceptual indexes that satisfy both axioms in the AD for the most cases.

Given this problem, a process is proposed in this work for establishing perceptual indexes based on the AD method to improve the design quality and efficiency. The development of the process can be divided into four steps. The adjectives that represent CAs are initially generated from past MRD/MRS. At the same time, the FRs are classified and generated from the past design specifications provided by a designer or design agency. Both the CAs and the FRs are used as the input to the quality function deployment (QFD) methodology to generate a design matrix. Based on the generated design matrix, corresponding transformations and optimizations are applied to the matrix to obtain a similar diagonal or lower triangle matrix that satisfies the independence axioms. Finally, the adjectives that represent customer attributes in the optimized design matrix are output. These adjectives can effectively guide the development of the MRD/MRS for similar products. The MRD and design specification data generated during the new product design can also be entered into the historical database to achieve iterative optimization.

\section{Literature Review}

\subsection{Axiomatic Design}

The AD principle, first raised by Suh (1990), provides a scientific and theoretical basis for designers to improve the design process. After years of development, the application of the AD principle has now extended to various fields including product design, system 
design, decision making, software design, and other aspects (Kulak, Cebi and Kahraman, 2010).

In Cheng (2012), a product platform design method in AD was proposed to improve the design efficiency and keep the costs low. Firstly, the FRs were classified into basic, expectable, and adjunctive type based on the Kano-model. Then, the AD principle was applied to the mapping process to obtain the design parameters based on the classification. Further, the relation degree among design parameters was identified through the design structure matrix to determine the basic, common, or individual platform parameters. Finally, a platform design of a drum brake was presented to show the effectiveness of the method.

Kahraman, Kaya, and Cebi (2009) proposed a method that comparatively used the AD and the analytic hierarchy process (AHP) for a renewable energy, fuzzy decisionmaking problem. In their fuzzy axiomatic design, the FRs for each criterion were predetermined by experts, and the final decision was obtained according to the information content values of each alternative's criteria based on the information axioms. Only the alternative that satisfies all of the criteria was selected as the final decision. The final result of using the AD method was the same as for the AHP, which showed that the $\mathrm{AD}$ can effectively deal with conflicting issues during the decisionmaking process.

Thielman and Ge (2006) applied the principle of AD to a large-scale engineering system. The FRs and their decomposition were first determined to form a qualitative design matrix. Based on this, the design matrix was transformed into a quantitative representation through a modelling method. Then, the requirements analysis and design matrix were both used to test the coupling degree of the design. Lastly, optimization and decoupling were done to improve the large-scale system. This method was also applied to a nuclear reactor system to verify its effectiveness in improving large-scale systems.

\subsection{Kansei Engineering}

KE was first developed in 1970 as a branch of engineering to study and fulfill customers' emotional and perceptual requirements. The method focused on analyzing customers' feelings on the material, color, or appearance of products through quantitative or statistical methods. After decades of development, KE achieved widespread adoption in various fields.

Jindo and Hirasago (1997) applied the theory of KE to the design of car interiors to satisfy customers' demands. Firstly, eight evaluation adjectives, such as "Elegant", "Playful", "Likable", were used to form a coordinate system; a semantic differential method was applied to subjectively evaluate the design. After this, the result was quantified, and a multivariate analysis was done to form the desired product according to the adjectives input. It was also indicated that the meaning of the evaluation adjectives for a certain product may be not the same in its related system evaluation. Dolgun and Köksal (2017) proposed a method that integrated KE and QFD to improve the product planning phase. The principle of KE was firstly used to identify customer requirements. Based on this, data collection was done in the form of Kansei words and 
the result was analyzed by empirical models. The priorities and targets of the customer and technical requirements were determined at last through the QFD methodology. This approach was applied to a yogurt product case, and the results showed that it had significantly improved the product planning phase.

Vieira et al. (2017) used KE to measure the users' subjective perceptions of rubber key pads. The data was collected by the semantic differential method according to the Kansei words from a survey. It was suggested from their results that the KE method was a robust method to evaluate the perceptual characteristics of products and Kansei words were closely related to the physical parameters. It was also indicated that the result of the Kansei experiment was affected by the age and gender of users.

\section{Method}

\subsection{The Generation of Customer Attributes and Functional Requirements}

As indicated by Suh (2011), a product's user requirements sometimes are hard to define; in some cases only fuzzy denotations are possible. To ensure the high cohesion of CAs in the user domain, the description of customer requirements can be replaced by Kansei adjectives to express the CAs more clearly and concisely.

In order to generate valuable Kansei adjectives that represent the CAs from a past MRD or MRS, word frequency statistics need to be initially performed. Basically, if a word has a high frequency of appearance in the MRD, then this word has a high relevance to the CAs. However, Zipf's law states that the frequency of a word's appearance is inversely proportional to its ranking in the frequency table, and the appearance of the most frequent word is approximately twice that of the second most frequent word (Zipf, 1972). This indicates that the words with most frequent appearance (e.g., "the", "of", "and") take up most of the MRD content while they do not contain any valuable information. Based on this, the "stop words" (e.g., prepositions, articles, conjunctions) are filtered out before carrying out the word frequency statistics; a stop word corpus can be adopted to support this filtering process. The words extracted from the word frequency statistics contain key information on potential customer requirements in an MRD. To transfer these keywords into adjectives that represent CAs, a semantic analysis based on a corpus can be used to improve the efficiency of this process. Since word frequency statistics have already extracted keywords, a simple lexical semantic analysis is adequate at this stage. It is indicated in Schneider's (2014) paper that WordNet can be a proper solution to basic lexical semantic analysis by mapping the inputs to synonym sets; in this work, each semantic category is linked to at least one lexical item. For this case, the filtered key words in an MRD are linked to synonymous adjectives through WordNet. The linked adjectives are provided by a design-related treebank or corpus, and the linked adjectives can be directly output as CAs. However, a design-related treebank or corpus are rare or not open source at present. Under such circumstance, a more extensive English Corpus such as WIKI50 Corpus (Vincze, Nagy and Berend, 2011) and Prague Dependency Treebank (Hajic,1998) can be adopted as a replacement; additional filtering for relevance can be applied before outputting the CAs. Figure 1 shows the generating process of CAs from the initial historical MRD. 


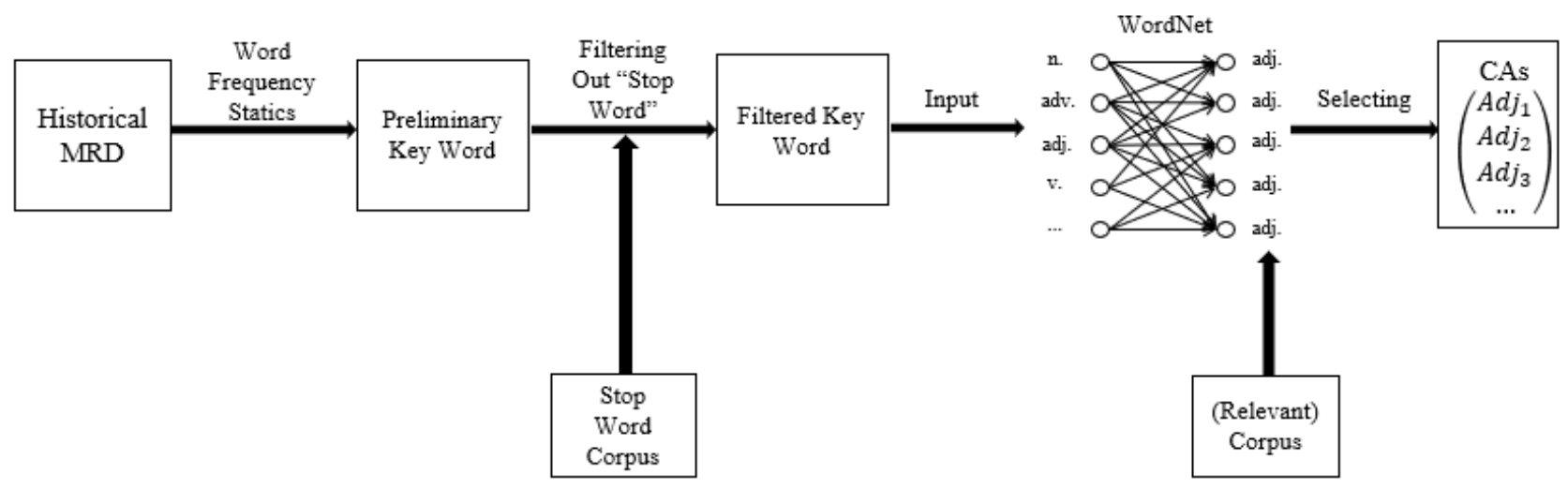

Figure 1. The flow diagram of the CAs generating process

Similarly, FRs can be generated from past design specifications provided by the designer or the design agency, and the FRs can be output in the form of entries. The same word frequency statistics and semantic analysis methods can also be used in this generating process. In Zisman and Kozlenkov's (2002) work, FRs are translated from natural language into URL sentences to conduct a consistency check with respect to the design specification. Such an approach can also be applied to this generating process to ensure its accuracy. The generating process and subsequent steps are illustrated in the flow diagram in Figure 2.

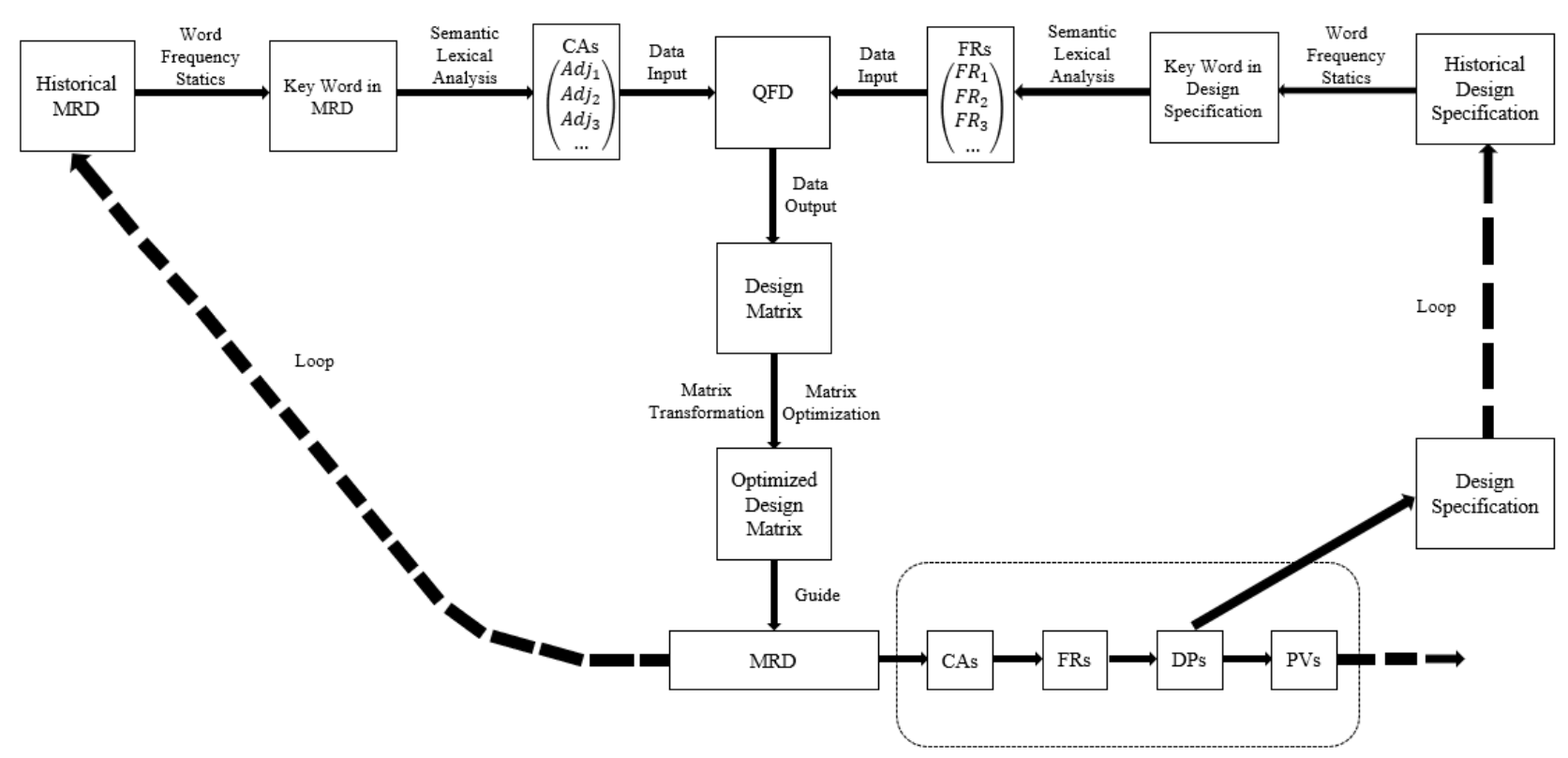

Figure 2. The flow diagram of the entire process

3.2 The Construction of the Design Matrix based on Quality Function Deployment The QFD methodology focuses on customer requirements and enhancing 
communication (Crawford, 1994). After extracting the adjectives of the CAs and the FRs entries, it can be used to generate a design structure matrix.

\begin{tabular}{|c|c|c|c|c|c|c|c|}
\hline $\begin{array}{c}\text { Functional } \\
\text { Requirement } \\
\text { (FRs) }\end{array}$ & 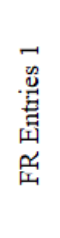 & 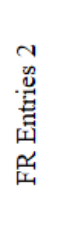 & 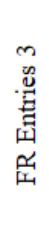 & $\vdots$ & & 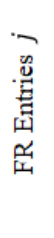 & 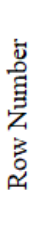 \\
\hline Kansei Adjectives 1 & $X_{11}$ & $X_{12}$ & $X_{13}$ & $\ldots$ & $\ldots$ & $X_{1 j}$ & 1 \\
\hline Kansei Adjectives 2 & $X_{21}$ & $X_{22}$ & $X_{23}$ & ... & ... & $X_{2 j}$ & 2 \\
\hline Kansei Adjectives 3 & $X_{31}$ & $X_{32}$ & $X_{33}$ & $\ldots$ & .. & $X_{3 j}$ & 3 \\
\hline$\ldots$ & $\ldots$ & $\ldots$ & $\ldots$ & $\ldots$ & .. & $\ldots$ & $\ldots$ \\
\hline$\ldots$ & $\ldots$ & $\ldots$ & $\ldots$ & $\ldots$ & $\ldots$ & $\ldots$ & $\ldots$ \\
\hline$\ldots$ & $\ldots$ & $\ldots$ & $\ldots$ & $\ldots$ & $\ldots$ & $\ldots$ & $\ldots$ \\
\hline Kansei Adjectives $i$ & $X_{i 1}$ & $X_{i 2}$ & $X_{i 3}$ & $\ldots$ & $\ldots$ & $X_{i j}$ & $i$ \\
\hline Column Number & 1 & 2 & 3 & $\ldots$ & $\ldots$ & $j$ & \\
\hline
\end{tabular}

Figure 3. The simplified $i \times j$ HOQ

As shown in Figure 3, a simplified house of quality (HOQ) is initially established. Compared to the common HOQ, the upper triangle roof and lower part are eliminated since they are not helpful to the generation of the design matrix. The collected Kansei adjectives and FR entries are entered into the table as the rows and columns. The element $X$ in the table represents the correlation of each FR to a corresponding CA; the scoring criteria are shown in Table 1. This step is performed by the relevant group of experts.

Table 1.

Evaluation criteria for the correlation degree:

\begin{tabular}{cc} 
Degree of Correlation & Score \\
\hline Strong Correlation & 5 \\
More Correlation & 4 \\
Common Correlation & 3 \\
Slight Correlation & 2 \\
Few Correlation & 1 \\
No Correlation & 0
\end{tabular}

Based on the table created, the elements in the HOQ can be organized into an $i \times j$ matrix T: 


$$
[T]=\left[\begin{array}{cccccc}
X_{11} & X_{12} & X_{13} & \ldots & \ldots & X_{1 j} \\
X_{21} & X_{22} & X_{23} & \ldots & \ldots & X_{2 j} \\
X_{31} & X_{32} & X_{33} & \ldots & \ldots & X_{3 j} \\
\ldots & \ldots & \ldots & \ldots & \ldots & \ldots \\
\ldots & \ldots & \ldots & \ldots & \ldots & \ldots \\
X_{i 1} & X_{i 2} & X_{i 3} & \ldots & \ldots & X_{i j}
\end{array}\right]
$$

According to the design function in the $\mathrm{AD}$ theory that explains the mapping process from FRs to design parameters (Suh, 2001):

$$
\{F R\}=[A]\{D P\}
$$

For an $i \times j$ design matrix $\mathrm{A}, F R_{i}$ can be expressed as:

$$
F R_{i}=\sum_{j=1}^{j} A_{i j} D P_{j}
$$

Based on this, the design function of the mapping process from the CAs to the FRs can be written as:

$$
\{C A\}=[B]\{F R\}
$$

For an $i \times j$ matrix $\mathrm{B}, C A_{i}$ can be written as:

$$
C A_{i}=\sum_{j=1}^{j} B_{i j} F R_{j}
$$

The $[\mathrm{B}]$ in the function represents the design structure matrix. Based on the matrix $\mathrm{T}$ generated from the QFD methodology, the design matrix [B] can be written as:

$$
[B]=\left[\begin{array}{cccccc}
B_{11} & B_{12} & B_{13} & \ldots & \ldots & B_{1 j} \\
B_{21} & B_{22} & B_{23} & \ldots & \ldots & B_{2 j} \\
B_{31} & B_{32} & B_{33} & \ldots & \ldots & B_{3 j} \\
\ldots & \ldots & \ldots & \ldots & \ldots & \ldots \\
\ldots & \ldots & \ldots & \ldots & \ldots & \ldots \\
B_{i 1} & B_{i 2} & B_{i 3} & \ldots & \ldots & B_{i j}
\end{array}\right]
$$

\subsection{Matrix Transformation and Optimization}

According to the independence axiom, an acceptable design matrix should be either a diagonal matrix or a lower triangular matrix; any other form of design matrix is called a full matrix (Suh, 2001). A full matrix does not satisfy the independence axioms, and its corresponding design is treated as a coupled design. Examples of these matrices are:

Diagonal Matrix: $\left[\begin{array}{l}C A_{1} \\ C A_{2} \\ C A_{3}\end{array}\right]=\left[\begin{array}{l}F R_{1} \\ F R_{2} \\ F R_{3}\end{array}\right]\left[\begin{array}{ccc}A_{11} & 0 & 0 \\ 0 & A_{22} & 0 \\ 0 & 0 & A_{33}\end{array}\right]$

Lower Triangle Matrix: $\left[\begin{array}{l}C A_{1} \\ C A_{2} \\ C A_{3}\end{array}\right]=\left[\begin{array}{l}F R_{1} \\ F R_{2} \\ F R_{3}\end{array}\right]\left[\begin{array}{ccc}A_{11} & 0 & 0 \\ A_{21} & A_{22} & 0 \\ A_{31} & A_{32} & A_{33}\end{array}\right]$

$$
\text { Full Matrix: }\left[\begin{array}{l}
C A_{1} \\
C A_{2} \\
C A_{3}
\end{array}\right]=\left[\begin{array}{l}
F R_{1} \\
F R_{2} \\
F R_{3}
\end{array}\right]\left[\begin{array}{ccc}
A_{11} & 0 & A_{13} \\
0 & A_{22} & 0 \\
0 & 0 & A_{33}
\end{array}\right]
$$

However, very few practical product designs can satisfy the definition of the diagonal 
matrix. Therefore, the optimization mainly concentrates on transforming a full matrix into (or close to) a lower triangle matrix. In this step, for different design matrices, the rearrangement, elimination, or addition of rows and columns is used to decouple the original matrix until it approaches a lower triangle matrix. The detailed process is listed in the following section.

\section{Case study}

4.1 Application of proposed approach

Portable chargers for consumer electronics have been adopted worldwide and are very popular products. However, some users complain about their heavy weights, limited energy capacities, and safety risks for some of the portable chargers on the market. Based on this, we list six major CAs and five FRs in the form of perceptual adjectives and entries by word frequency statistics and semantic lexical analysis on the historical product's MRD and design specification. These are presented in Table 2.

Table 2.

The collected CAs and FRs from the historical product MRD and design specification

\begin{tabular}{ccc} 
& Customer Attributes (CAs) & Functional Requirement (FRs) \\
\hline 1 & Cheap & Streamlined Appearance \\
3 & Industrial Feeling & Ergonmic Design \\
4 & Long Battery Life & High Density Cell \\
4 & Light & High Temperature Tolerance \\
5 & Handy operated & Material \\
6 & Safe &
\end{tabular}

In the next step, the collected CAs and FRs were then input into the HOQ table as the rows and columns. According to evaluation criterion, the average correlation values of each FR to a corresponding CA were provided by a group of experts and engineers with relevant experience. The HOQ table for a portable charger design is shown in Figure 4. 


\begin{tabular}{|c|c|c|c|c|c|c|c|}
\hline $\begin{array}{c}\text { Functional } \\
\text { Requirement } \\
\text { (FRs) }\end{array}$ & 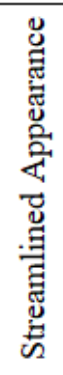 & 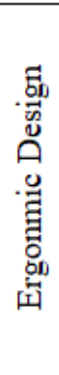 & 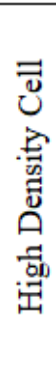 & 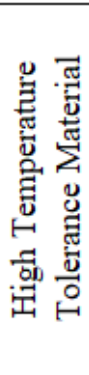 & 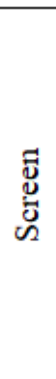 & 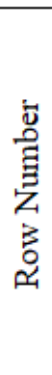 & 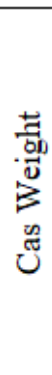 \\
\hline Cheap & 1 & 1 & 3 & 3 & 5 & 1 & 5 \\
\hline Industrial Feeling & 5 & 3 & 0 & 0 & 3 & 2 & 3 \\
\hline Long Battery Life & 1 & 0 & 5 & 0 & 0 & 3 & 5 \\
\hline Light & 0 & 0 & 3 & 1 & 0 & 4 & 3 \\
\hline Handy operated & 0 & 5 & 0 & 0 & 1 & 5 & 1 \\
\hline Safe & 1 & 0 & 3 & 5 & 0 & 6 & 5 \\
\hline Column Number & 1 & 2 & 3 & 4 & 5 & & \\
\hline
\end{tabular}

Figure 4. The HOQ table for a portable charger design

The corresponding element in the above HOQ table can be organized into a $6 \times 5$ matrix T:

$$
[T]=\left[\begin{array}{lllll}
1 & 1 & 3 & 3 & 5 \\
5 & 3 & 0 & 0 & 3 \\
1 & 0 & 5 & 0 & 0 \\
0 & 0 & 3 & 1 & 0 \\
0 & 5 & 0 & 0 & 1 \\
1 & 0 & 3 & 5 & 0
\end{array}\right]
$$

According to the equations (1)-(4), the design matrix B for this case can be written as:

$$
[B]=\left[\begin{array}{ccccc}
B_{11} & B_{12} & B_{13} & B_{14} & B_{15} \\
B_{21} & B_{22} & 0 & 0 & B_{25} \\
B_{31} & 0 & B_{33} & 0 & 0 \\
0 & 0 & B_{43} & B_{44} & 0 \\
0 & B_{52} & 0 & 0 & B_{55} \\
B_{61} & 0 & B_{63} & B_{64} & 0
\end{array}\right]
$$

It can be easily found that matrix B is a full matrix because the elements in its upper triangle region are not all equal to zero, which indicates its corresponding design is a coupled design. Therefore, it is necessary to transform matrix B into a diagonal or lower triangle matrix in order to obtain an acceptable design. After the rearrangement to with respect to its rows, the transformed design matrix $B^{\prime}$ and $T^{\prime}$ can be written as: 


$$
\left[B^{\prime}\right]=\left[\begin{array}{ccccc}
B^{\prime}{ }_{21} & B^{\prime}{ }_{22} & 0 & 0 & B^{\prime}{ }_{25} \\
0 & B^{\prime}{ }_{52} & 0 & 0 & B^{\prime}{ }_{55} \\
B^{\prime}{ }_{31} & 0 & B^{\prime}{ }_{33} & 0 & 0 \\
B^{\prime}{ }_{61} & 0 & B^{\prime}{ }_{63} & B^{\prime}{ }_{64} & 0 \\
B^{\prime}{ }_{11} & B^{\prime}{ }_{12} & B^{\prime}{ }_{13} & B^{\prime}{ }_{14} & B^{\prime}{ }_{15} \\
0 & 0 & B^{\prime}{ }_{43} & B^{\prime}{ }_{44} & 0
\end{array}\right] \quad\left[T^{\prime}\right]=\left[\begin{array}{ccccc}
5 & 3 & 0 & 0 & 3 \\
0 & 5 & 0 & 0 & 1 \\
1 & 0 & 5 & 0 & 0 \\
1 & 0 & 3 & 5 & 0 \\
1 & 1 & 3 & 3 & 5 \\
0 & 0 & 3 & 1 & 0
\end{array}\right]
$$

The new corresponding transformed HOQ table is:

\begin{tabular}{|c|c|c|c|c|c|c|c|}
\hline $\begin{array}{c}\text { Functional } \\
\text { Requirement } \\
\text { (FRs) }\end{array}$ & 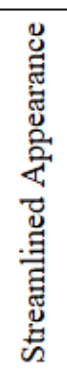 & 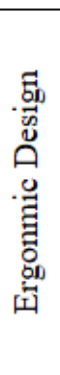 & 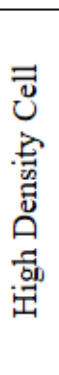 & 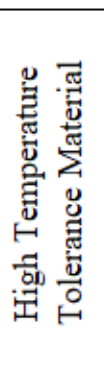 & $\begin{array}{l}\text { : } \\
\text { ญ. } \\
\text { ผ }\end{array}$ & 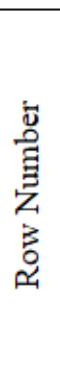 & 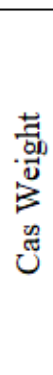 \\
\hline Industrial Feeling & 5 & 3 & 0 & 0 & 3 & 1 & 3 \\
\hline Handy operated & 0 & 5 & 0 & 0 & 1 & 2 & 1 \\
\hline Long Battery Life & 1 & 0 & 5 & 0 & 0 & 3 & 5 \\
\hline Safe & 1 & 0 & 3 & 5 & 0 & 4 & 5 \\
\hline Cheap & 1 & 1 & 3 & 3 & 5 & 5 & 1 \\
\hline Light & 0 & 0 & 3 & 1 & 0 & 6 & 3 \\
\hline Column Number & 1 & 2 & 3 & 4 & 5 & & \\
\hline
\end{tabular}

Figure 5. The HOQ table after the transformation

It can be noticed that the existence of the 6th row, which represents the CA "Light", strongly inhibits the transformation of the design matrix into a lower triangle matrix. Considering that it is only related to two entries of FRs and their importance score is relatively low, this CA row is eliminated to optimize the transformed matrix. The optimized design matrix $B^{\prime \prime}$ and $T^{\prime \prime}$ can be written as:

$\left[B^{\prime \prime}\right]=\left[\begin{array}{ccccc}B^{\prime \prime}{ }_{21} & B^{\prime \prime}{ }_{22} & 0 & 0 & B^{\prime \prime}{ }_{25} \\ 0 & B^{\prime \prime}{ }_{52} & 0 & 0 & B^{\prime \prime}{ }_{55} \\ B^{\prime \prime}{ }_{31} & 0 & B^{\prime \prime}{ }_{33} & 0 & 0 \\ B^{\prime \prime}{ }_{61} & 0 & B^{\prime \prime}{ }_{63} & B^{\prime \prime}{ }_{64} & 0 \\ B^{\prime \prime}{ }_{11} & B^{\prime \prime}{ }_{12} & B^{\prime \prime}{ }_{13} & B^{\prime \prime}{ }_{14} & B^{\prime \prime}{ }_{15}\end{array}\right] \quad\left[T^{\prime \prime}\right]=\left[\begin{array}{ccccc}5 & 3 & 0 & 0 & 3 \\ 0 & 5 & 0 & 0 & 1 \\ 1 & 0 & 5 & 0 & 0 \\ 1 & 0 & 3 & 5 & 0 \\ 1 & 1 & 3 & 3 & 5\end{array}\right]$ 
The corresponding HOQ table after the optimization is:

\begin{tabular}{|c|c|c|c|c|c|c|c|}
\hline $\begin{array}{c}\text { Functional } \\
\text { Requirement } \\
\text { (FRs) }\end{array}$ & 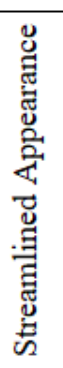 & 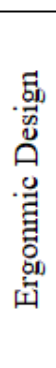 & 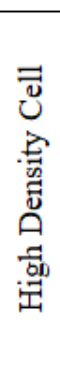 & 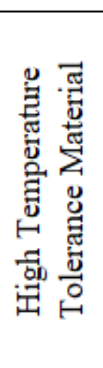 & 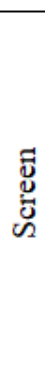 & 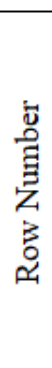 & 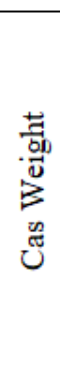 \\
\hline Industrial Feeling & 5 & 3 & 0 & 0 & 3 & 1 & 3 \\
\hline Handy operated & 0 & 5 & 0 & 0 & 1 & 2 & 1 \\
\hline Long Battery Life & 1 & 0 & 5 & 0 & 0 & 3 & 5 \\
\hline Safe & 1 & 0 & 3 & 5 & 0 & 4 & 5 \\
\hline Cheap & 1 & 1 & 3 & 3 & 5 & 5 & 1 \\
\hline Column Number & 1 & 2 & 3 & 4 & 5 & & \\
\hline
\end{tabular}

Figure 6. The HOQ table after the optimization

After the transformation and elimination of the CA rows, the optimized design matrix $B^{\prime \prime}$ has become more similar to a lower triangle matrix. There are still three elements above the diagonal that are nonzero; however, their corresponding importance scores are relatively low. Therefore, ignoring these nonzero values can be acceptable, and the output of the five adjectives that represent the CAs as a reference for guiding the MRD is performed directly. The MRD and design specification in the next design can also be recorded in a historical database to achieve iterative optimization and make the entire process become a closed loop (refer to Figure 2).

\subsection{Discussion}

After going through the complete process including word frequency statistics, semantic analysis, QFD, matrix construction and optimization, the obtained perceptual indexes satisfy the independence axioms and effectively guide the practical product design. However, during the final design matrix optimization process, the direct output of the CAs from the matrix $B^{\prime \prime}$ (Section 4.1) should have the precondition that the values of the coupling/non-zero elements above the diagonal are relatively low. This means that such a process may become inapplicable when, for example, the coupling elements above the diagonal are equal to five or most are greater than one. Therefore, in order to make this process broadly applicable, a method is necessary to make these coupling elements approach zero.

In Thielman and Ge's (2006) paper, the reangularity semiangularity ratio (R/S) analysis method was used to measure and reduce the coupling degree of a large-scale system design based on the AD principles. However, this method is for non-linear multi-FR 
designs; the FR-DP mapping process in the $\mathrm{AD}$ approach can be directly used in this case.

In Zhang et al. (2008), two methods including clustering and auxiliary functions were introduced to eliminate the coupling elements above the diagonal in the design matrix. The first method clusters the coupling rows with other rows into new modules and then further eliminates the coupling through brainstorming activities. The second method decouples the design matrix by adding an extra auxiliary row into the bottom of the matrix to support fixing the coupling elements through a knowledge base or an expert review. Both of these methods can be good solutions to eliminate the coupling elements above the diagonal when the corresponding value exceeds expectations. However, these two decoupling methods contain subjective factors which may influence the accuracy of the final result to some extent.

\section{Conclusions and Future work}

Based on the problem of mapping customers' need from the user domain to the functional domain in $\mathrm{AD}$, this paper develops a process for establishing perceptual indexes based on AD to improve design quality and efficiency. To obtain valuable CRs and FRs from the MRD and design specification, semantic analysis and word frequency statistics are used as the first step. Secondly, the QFD methodology is adopted to generate the design matrix. In order to obtain the CAs that satisfy the independence axioms, steps to transform and optimize are applied to the design matrix generated by the QFD. Finally, the qualified adjectives are output as the reference for guiding the MRD. The MRD and design specification in the next design can also be entered into a historical database to achieve iterative optimization.

In the future, the following interesting and valuable research directions are going to be investigated:

- Investigate the application of the $\mathrm{R} / \mathrm{S}$ ratio analysis method into the CA-FR mapping process to measure the coupling degree of the design structure matrix before and after the optimization. This measurement has the potential to support the systematic, quantified elimination or reduction of the coupling degree.

- Establish or enlarge the design-related corpus to eliminate the extra filtering steps applied after using WordNet to improve the accuracy and efficiency of the output. 


\section{References}

Cheng, X. (2012). Functional Requirements Analysis-Based Method for Product Platform Design in Axiomatic Design. Journal of Digital Information Management, 10(5), 312-319.

Crawford, M. (1994). How Puritan-Bennett used the house of quality John R. Hauser, Sloan Management Review (Spring 1993), pp. 61-70. Journal of Product Innovation Management, 11(3), p.271.

Dolgun, L. and Köksal, G. (2017). Effective use of quality function deployment and Kansei engineering for product planning with sensory customer requirements: A plain yogurt case. Quality Engineering, 30(4), pp.569-582.

Hajic, J. (1998). Building a syntactically annotated corpus: The prague dependency treebank. Issues of Valency and Meaning. Studies in Honor of Jarmila Panevová, pp.1219.

Jindo, T. and Hirasago, K. (1997). Application studies to car interior of Kansei engineering. International Journal of Industrial Ergonomics, 19(2), pp.105-114.

Samuel, F. (2009). Specification and Analysis of Requirements Negotiation Strategy in Software Ecosystems.IWSECO@ICSR,pp.22-23.

Kahraman, C., Kaya, İ. and Cebi, S. (2009). A comparative analysis for multiattribute selection among renewable energy alternatives using fuzzy axiomatic design and fuzzy analytic hierarchy process. Energy, 34(10), pp.1603-1616.

Kulak, O., Cebi, S. and Kahraman, C. (2010). Applications of axiomatic design principles: A literature review. Expert Systems with Applications, 37(9), pp.6705-6717.

Schneider, N. (2014). Lexical semantic analysis in natural language text. Unpublished Doctoral Dissertation, Carnegie Mellon University, pp.8-10.

Suh, N. and Sekimoto, S. (1990). Design of Thinking Design Machine. CIRP Annals, 39(1), pp.145-148.

Suh, N. (2001). Axiomatic design. New York, N.Y.: Oxford University Press, pp.9-10, 14-18.

Thielman, J. and Ge, P. (2006). Applying axiomatic design theory to the evaluation and optimization of large-scale engineering systems. Journal of Engineering Design, 17(1), pp.1-16.

Vieira, J., Osório, J., Mouta, S., Delgado, P., Portinha, A., Meireles, J. and Santos, J. 
(2017). Kansei engineering as a tool for the design of in-vehicle rubber keypads. Applied Ergonomics, 61, pp.1-11.

Vincze, V., Nagy, I. and Berend, G. (2011). Multiword expressions and named entities in the Wiki50 corpus. Proceedings of the International Conference Recent Advances in Natural Language Processing 2011, pp.289-295.

Zhang, G., Dunbing, T. and Sheng,D.(2008). Research on Integration of Axiomatic Design Matrix and Design Structure Matrix. Machine Design and Research, 24(5), pp.16-18.

Zipf, G. (1972). Human behaviour and the principle of least effort. New York: Hafner Publishing.

Zisman, A. and Kozlenkov, A. (2002). Are their design specifications consistent with our requirements?. Proceedings IEEE Joint International Conference on Requirements Engineering, pp.145-154. 